Mon. Not. R. Astron. Soc. 000, 1 13 (2010) Printed 22 June $2018 \quad$ (MN LATEX style file v2.2)

\title{
The Planetary Nebulae Population in the Central Regions of M32: the SAURON view
}

\author{
Marc Sarzi ${ }^{1 \star}$, Gary Mamon $^{2}$, Michele Cappellari $^{3}$, Eric Emsellem $^{4,5}$, Roland Bacon $^{5}$, \\ Roger L. Davies, P. Tim de Zeeuw ${ }^{4,6}$ \\ ${ }^{1}$ Centre for Astrophysics Research, University of Hertfordshire, College Lane, Hatfield, Herts, AL10 9AB, UK \\ ${ }^{2}$ Institut d'Astrophysique de Paris, 98 bis Bd. Arago, 75014 Paris, France \\ ${ }^{3}$ Sub-Dept of Astrophysics, Dept of Physics, University of Oxford, Denys Wilkinson Building, Keble Road, Oxford, OX1 3RH, UK \\ ${ }^{4}$ European Southern Observatory, Karl-Schwarzschild-Str 2, 85748 Garching, Germany \\ ${ }^{5}$ Centre de Recherche Astronomique de Lyon, 9 Avenue Charles André, 69230 Saint Genis Laval, France \\ ${ }^{6}$ Sterrewacht Leiden, Universiteit Leiden, Postbus 9513, 2300 RA Leiden, The Netherlands
}

22 June 2018

\begin{abstract}
Extragalactic Planetary Nebulae (PNe) are not only useful as distance signposts or as tracers of the dark-matter content of their host galaxies, but constitute also good indicators of the main properties of their parent stellar populations. Yet, so far, the properties of PNe in the optical regions of galaxies where stellar population gradients can be more extreme have remained largely unexplored, mainly because the detection of PNe with narrow-band imaging or slit-less spectroscopy is considerably hampered by the presence of a strong stellar background. Integral-field spectroscopy (IFS) can overcome this limitation, and here we present a study of the PN population in the nearby compact elliptical M32. Using SAURON data taken with just two 10-minutes-long pointings we have doubled the number of known PNe within the effective radius of M32, detecting PNe five times fainter than previously found in narrowband images that collected nearly the same number of photons. We have carefully assessed the incompleteness limit of our survey, and accounting for it across the entire range of luminosity values spanned by our detected PNe, we could conclude despite having at our disposal only 15 sources that the central PNe population of M32 is consistent with the generally adopted shape for the PNe Luminosity Function and its typical normalization observed in early-type galaxies. Furthermore, owing to the proximity of M32 and to ultraviolet images taken with the Hubble Space Telescope, we could identify the most likely candidates for the central star of a subset of our detected PNe and conclude that these stars are affected by substantial amounts of circumstellar dust extinction, a finding that could reconcile the intriguing discrepancy previously reported in M32 between the model predictions and the observations for the later stages of stellar evolution. Considering the modest time investment on a $4 \mathrm{~m}$-class telescope that delivered these results, this study illustrates the potential of future IFS investigations for the central PNe population of early-type galaxies, either with existing SAURON data for many more, albeit more distant, objects, or from campaigns that will use the future generations of integral-field spectrographs that will be mounted on 8m-class telescopes, such as MUSE on the Very Large Telescope.
\end{abstract}

Key words: galaxies: elliptical and lenticular - galaxies: stellar content - galaxies: individual: M32 - ISM: planetary nebulae: general - stars: AGB and post-AGB

\section{INTRODUCTION}

In the field of extra-galactic astronomy, Planetary Nebulae $(\mathrm{PNe})$ are perhaps regarded mostly either as useful indicators for the distance of their galactic hosts (Ciardullo et al. 1989;
Jacoby, Ciardullo, \& Ford 1990; Jacoby et al. 1992), thanks to the almost universal - though not fully understood - shape of their luminosity function (PNLF, generally in the [O III] $\lambda 5007$ emission), or as tracers of the gravitational potential in the outskirts of galaxies Romanowsky et al. 2003; Douglas et al. 2007). Yet, as summarised in the review of Ciardullo (2006), PNe can also be used as probes of their parent stellar population. For the clos-

\footnotetext{
* E-mail :m.sarzi@herts.ac.uk
} 
est or brightest $\mathrm{PNe}$, the detection of critical but weak diagnostic emission lines such as [O III] $\lambda 4363$ allows to directly measure the temperature of the ionised-gas and thus the metallicity of PNe, which in turn makes it possible to constrain the chemical enrichment history of their host galaxy (e.g., Richer, Stasińka, \& McCall 1999; Jacoby \& Ciardullo 1999; Dopita et al. 1997). In more distant galaxies, it is still possible to place useful constraints on the Oxygen abundance of PNe by using brighter lines such a [O III] $\lambda 5007, \mathrm{H} \beta,[\mathrm{N} \mathrm{II}] \lambda \lambda 6548,6584, \mathrm{H} \alpha$ or [S II] $\lambda \lambda 6713,6731$, or to investigate the star-formation history of a galaxy by studying the shape and normalisation of the PNLF (e.g., Marigo et al. 2004; Schönberner et al. 2007; Méndez et al. 2008). In fact, understanding the origin of the PNLF is a puzzle that, once solved, promises to reveal new clues on the late stages of stellar evolution and on the formation of PNe themselves (e.g., Ciardullo et al.2005; Buzzoni, Arnaboldi, \& Corradi 2006).

In this context it is important to note that most known extragalactic PNe have been found in the outskirts of their hosts, whereas the PNe population of the optical regions of galaxies remains largely unexplored. Most PNe studies have indeed been carried out through narrow-band imaging (e.g., Ciardullo et al. 1989) or slit-less spectroscopy (e.g., Douglas et al. 2007), where the detection of PNe is considerably hampered by the presence of a stellar background. Yet, it is in central regions of galaxies where stellar population gradients can be more extreme and where the stellar ages, metallicities and element abundances can be the most diverse between different galaxies. Integral-field spectroscopy can overcome the previous instrumental limitations since it allows for the careful modelling of a galaxy's integrated stellar spectrum, and this paper aims to demonstrate the potential of IFS for studying the properties on PNe in the optical regions of galaxies using SAURON observations for the compact elliptical M32 (NGC221), our closest early-type galaxy.

This work is organized as follows. In $\$ 2$ we briefly review the acquisition and reduction of the SAURON data for M32. In 3 we detail how we optimised the extraction of the nebular emission in the SAURON data and how we proceeded to identify and measure the [O III] 55007 flux of the PNe in M32. In 4 we present our main results, assessing in particular whether our data are consistent with the generally adopted shape for PNLF and the value for its normalisation that is most commonly observed in early-type galaxies. In 5 we discuss these findings by linking them to the known properties of the stellar population of M32, and in particular to the apparent dearth of post-asymptotic giant branch stars that was reported by Brown et al. (2000). Finally, in 6 we draw our conclusions and consider some future prospects for more IFS studies of the PNe populations in the central regions of galaxies.

\section{OBSERVATIONS AND DATA REDUCTIONS}

M32 (NGC221) was one of the special objects that were observed over the course of the SAURON representative survey (de Zeeuw et al. 2002), and more specifically during the last run of that observing campaign at the $4 \mathrm{~m}$ William Herschel Telescope and following the installation of a new volume phase-holographic grating (Emsellem et al. 2004). The central regions of M32 were observed with two offset pointings during 600s for each exposure, while using the low-resolution mode of SAURON that gives a field of view of $33^{\prime \prime} .0 \times 44^{\prime \prime} .0$ fully sampled by $0 . .94 \times 0 . .94$ square lenses (for more details on the instrument see Bacon et al. 2001). The data from each pointing were reduced similarly to the

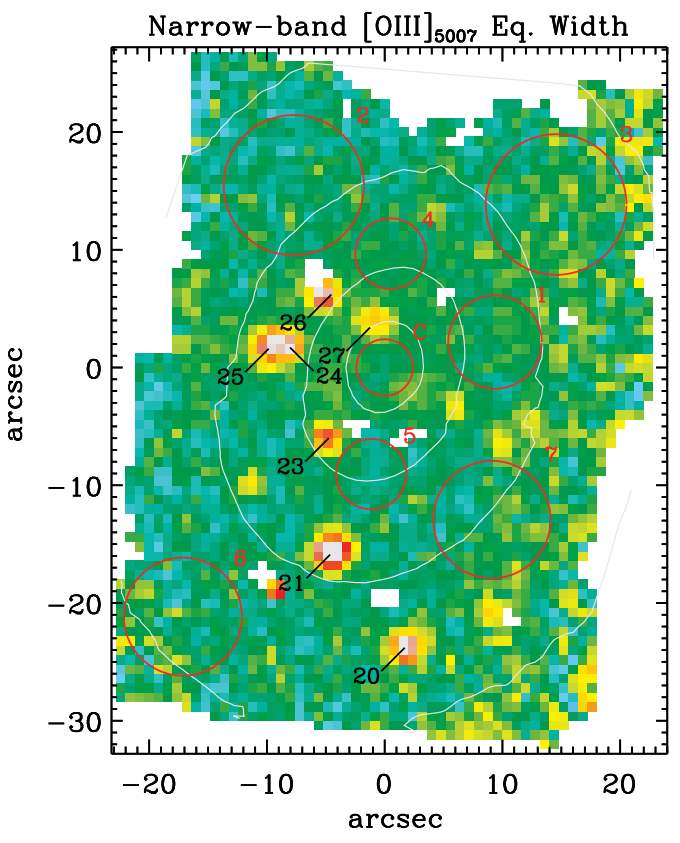

Figure 1. Map of the equivalent width (EW) of the estimated [O III] $\lambda 5007$ flux from narrow-band imaging based on the SAURON data. The flux in the $[\mathrm{O}$ III] region is measured through a $2.5 \AA$-wide spectral window centred on the expected position of [O III] given the systemic velocity of $V_{\text {sys }}=-197 \mathrm{~km} \mathrm{~s}^{-1}$ for M32, whereas the flux of the stellar continuum is computed using the mean spectral energy density across the entire spectrum times the same $2.5 \AA$ wavelength interval. The difference between these two flux measurements gives an estimate of the [O III] flux, which once divided by the mean spectral energy density in the stellar continuum leads to the mapped EW values. Isolated patches of positive EW values could originate from the unresolved [O III] emission of PNe, and in fact the brightest and most circular sources correspond to the $7 \mathrm{PNe}$ that were already identified in the optical regions of M32 by Ciardullo et al. (1989, shown and labelled in black). The red circles delimit galactic regions that are most likely devoid of any nebular emission and where the SAURON spectra were added up to obtain spectra of very high quality. In turn, these high-quality spectra were used to derive the optimal templates that we adopted to describe the stellar continuum in our data (see text for more details).

data obtained for the objects of the main SAURON sample (see Emsellem et al. 2004; Falcón-Barroso et al. 2006), and the resulting datacubes were merged and resampled in $0.0^{\prime \prime} 8 \times 0.0^{\prime \prime} 8$ spatial elements each corresponding to spectra with a final spectral resolution of $4.2 \AA$ (FWHM).

The present SAURON data for M32 were already used to extract the stellar kinematics that was modeled by Cappellari et al. (2006) and the only difference with the data used in that work and other papers of the SAURON project is that here we did not perform any Voronoi spatial binning (Cappellari \& Copin 2003). This was done to avoid swamping the signal of the weaker PNe against an increased stellar background, and to allow for a more consistent analysis of the flux distribution from each of the unresolved PN that we may detect. 


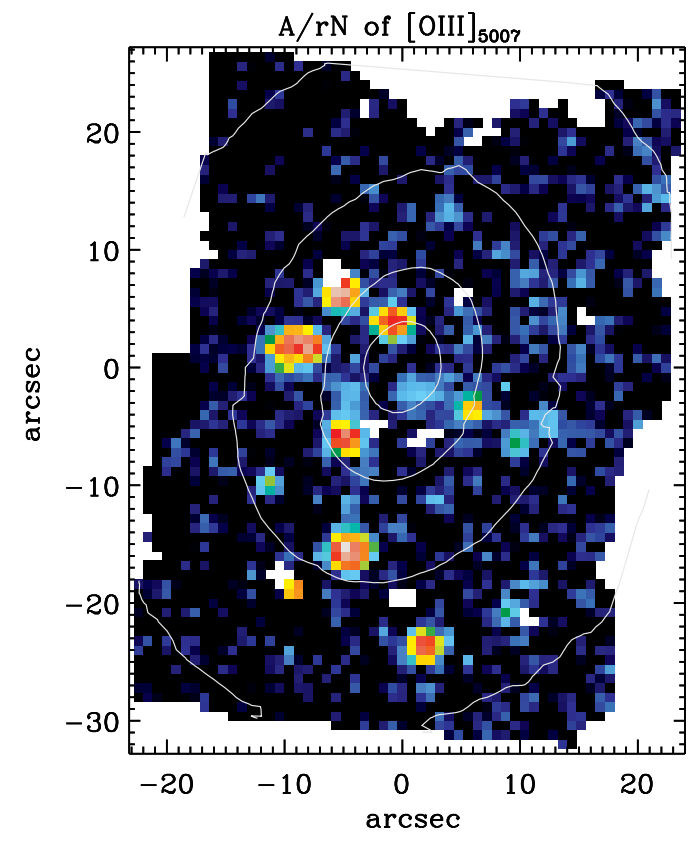

Figure 2. Map of the values of the $A / r N$ ratio of the [O III] $\lambda 5007$ emission measured in the SAURON spectra. The $A / r N$ values are shown in a logarithmic scale and dark-blue bins identify regions where $A / r N<3$ and the [O III] line is not formally detected. Several of the weak positive patches in Fig. 1 correspond here to circular sources with gently declining $A / r N$ profiles as would be expected from atmospheric scattering of the [O III] emission of an unresolved PN. This is not the case for the rectangular patch approximately $20^{\prime \prime}$ to south and east of the centre, which is a spurious measurement where in fact no [O III] $\lambda 4959$ emission is detected along with the larger $[\mathrm{O} I I I] \lambda 5007$ line.

\section{DATA ANALYSIS}

\subsection{Emission-line Measurements}

In order to identify $\mathrm{PNe}$ in M32 and measure their flux in the [O III $] \lambda 5007$ line we first need to separate as accurately as possible the stellar and nebular contribution to each of the SAURON spectra that sample the central regions of this galaxy. For this purpose we used the method of Sarzi et al. (2006, hereafter Paper V following the notation of the SAURON project) whereby a set of stellar templates and Gaussian emission lines are fitted simultaneously to the spectre 1 , while following also the approach of Sarzi et al. (2010) to further improve the match to the stellar continuum and ensure that the ionised-gas emission is extracted from the subtraction of a physically motivated stellar model. This is achieved by using, instead of standard template libraries based on stellar spectra or single-age stellar population models, a more appropriate set of empirical templates that are constructed while matching a number of high-quality SAURON spectra extracted from regions in the target galaxy where no ionised-gas emission is found.

Fig. 1 shows how we identified such emission-line free regions in M32 using an [O III] narrow-band image made from

1 In practice this is achieved by using the IDL code GANDALF (available at http://star-www.herts.ac.uk/ sarzi and the stellar Kinematics extracted with the pixerfitting IDL code pPXF Cappellari \& Emsellem 2004, http: //www-astro.ox.ac.uk/ mxc/idl

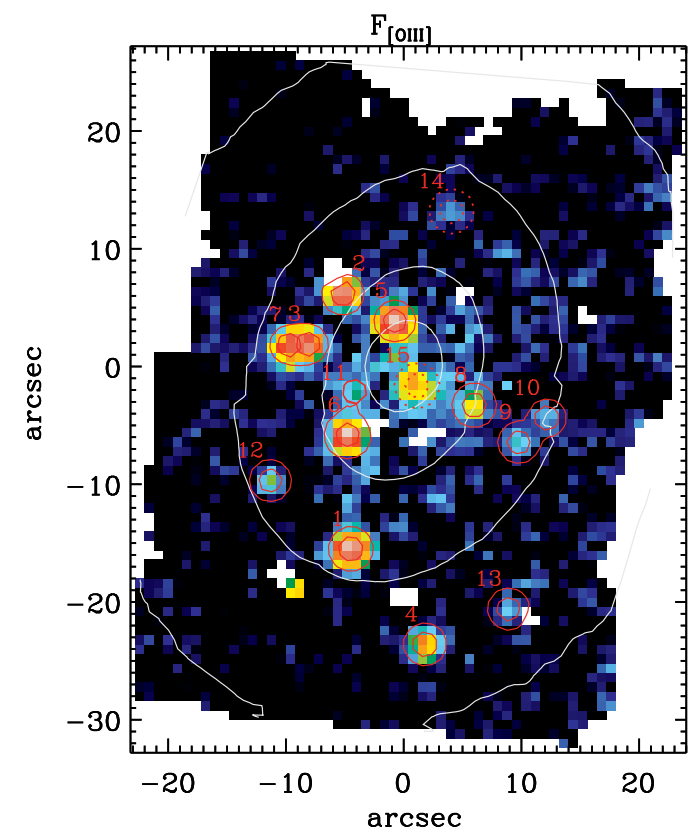

Figure 3. Map of the flux of the [O III] $\lambda 5007$ emission from M32, in $10^{-16} \mathrm{erg} \mathrm{s}^{-1} \mathrm{~cm}^{-2} \operatorname{arcsec}^{-2}$ and in a logarithmic scale, together with the photometric measurements of both firmly and marginally detected PNe. Each of the PNe is labelled and delineated by red contours (in solid and dotted lines for solid and marginal detections, respectively) corresponding to the best-fitting Gaussian model to their observed flux distribution, which is used to compute their total [O III] flux $F_{5007}$ and corresponding detection limit (see text). The inner contour around each PNe shows the half-peak flux level of the Gaussian model, thus corresponding to a circle a FWHM in diameter, whereas the second contour shows the region containing $90 \%$ of the flux of the model around each PN, or of both the Gaussians models in the case of blended PNe sources.

the SAURON data themselves (see the caption of Fig. 1 for details). Each of the spectra extracted from the circular apertures shown in Fig. 1 was fitted with the pixel-fitting code of Cappellari \& Emsellem (2004), over the full wavelength range of the SAURON data and using the entire MILES template library of Sánchez-Blázquez et al. (2006). Both the quality of such aperture spectra and of our fit to them is very high, with values for the ratio between the median level of the spectra $(S)$ and the average level of the fit residuals (residual noise, $r N$ ) ranging from 100 to over 400 , with $S / r N \sim 300$ on average. The weights assigned to the MILES stellar templates during each of these fits were then used to combine the MILES spectra into optimal templates that, owing to the excellent quality of our fit, can be in practice regarded as M32 spectra deconvolved from the line-of-sight kinematical broadening. Fig. 1 also illustrates how our emission-free apertures are evenly spread over the SAURON field of view, which ensures that their corresponding optimal templates can account for the presence of stellar population gradients (Rose et al. 2005) when they are used to match each of the single spectra sampling the central regions of M32.

Fig. 11also locates the PNe found in the narrow-band survey of Ciardullo et al. (1989), and reveals already the presence of weaker and isolated peaks of $5007 \AA$ flux that may also originate from the unresolved [O III] emission of PNe. If this is the case, then the nebular spectra observed where the flux of an unresolved PN is scattered by the atmospheric point-spread function (PSF) should 

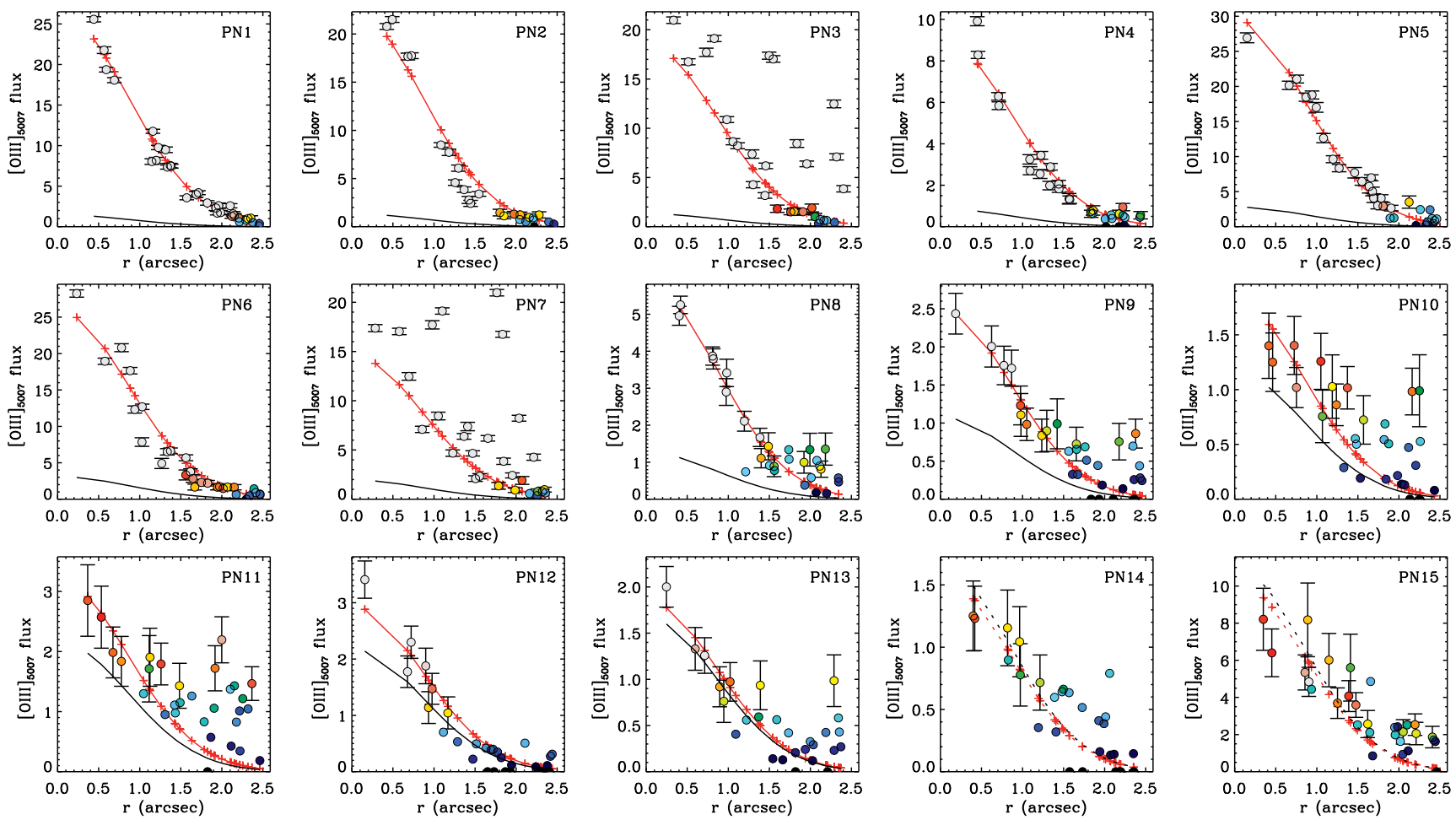

Figure 4. Radial profiles of the flux of the [O III] $\lambda 5007$ line (in $10^{-16} \mathrm{erg} \mathrm{s}^{-1} \mathrm{~cm}^{-2} \operatorname{arcsec}^{-2}$ ) of the PNe sources shown in Fig. 3] along with their corresponding best-fitting Gaussian model for the flux distribution (red lines, models are resampled in the SAURON 0 !' $8 \times 0$ ". 8 bins). Radial distances are computed from the center of the Gaussian models and the data points are color coded according to the value of the $A / r N$ ratio. Colors change from blue to red for increasing values of $A / r N$ till they saturate to white for $A / r N>6$. Green corresponds to the detection threshold of $A / r N=3$, above which the data points are also plotted together with error bars for the [O III] flux. The black lines show the flux of the Gaussian model that would fit the data if these were rescaled until the minimum value of $A / r N$ within the FWHM (for a radius of 0. '96) of the model reaches the threshold of $A / r N=3$. When integrated, the best-fitting and rescaled Gaussian models yield the total flux $F_{5007}$ of each PN source and its corresponding detection limit, respectively. The model profiles for PNe 14 and 15 are shown with dashed lines to indicate that these are doubtful sources according the previous definition of the detection limit. The radial profiles extend out to a radius $r=3 \sigma_{\mathrm{PSF}}$, where $\sigma_{\mathrm{PSF}}$ was determined from the fit of the brightest source PN 1 . PNe 3 and 7, 6 and 11,9 and 10 have been modeled simultaneously.

be characterised by large values of the $[\mathrm{O}$ III $] / \mathrm{H} \beta$ ratio, virtually no $[\mathrm{N}$ I $] \lambda \lambda 5197,5200$ emission, and by very narrow lines that would not be resolved in the SAURON spectra. The standard fitting strategy of Paper V, whereby the [O III] doublet is fitted first and the $\mathrm{H} \beta$ flux is subsequently extracted holding the $\mathrm{H} \beta$ line profile to that of the [O III] lines, is therefore appropriate for isolating the emission of $\mathrm{PNe}$, and here we only further imposed a fixed width on the [O III] lines, for an observed velocity dispersion of $108 \mathrm{~km} \mathrm{~s}^{-1}$ that corresponds to the spectral resolution of the SAURON data ( $(2)$.

Fig. 2 shows the map of the ratio between the best-fitting Gaussian amplitude $A$ for the [O III] $\lambda 5007$ line and the noise level $r N$ in the fit residuals. In Paper $\mathrm{V}$ we concluded that a minimum value of $A / r N=4$ was required to detect [O III] emission, although this was while letting the width of the lines free to vary. Since instead here we have fixed the [O III] line width, our fits involve one less free parameter and we can measure unbiased [O III] fluxes also down to a $A / r N=3$ threshold, similarly to the case of $\mathrm{H} \beta$ line measurements of Paper $\mathrm{V}$ when the [O III] line profile was imposed on the $\mathrm{H} \beta$ line. With such a detection limit, Fig. 2] confirms the presence of several isolated weak patches of detected [O III] emission beside the PNe of Ciardullo et al. (1989), and shows the absence of a diffuse ionised-gas component that is otherwise commonly found in early-type galaxies (Paper V).

\subsection{PNe Detection and Flux Measurements}

To measure the flux of any known or possible PNe in the SAURON field-of-view, and at the same time establish whether a weak patch of [O III] emission is indeed consistent with the flux distribution expected from an unresolved PN, we fitted each of the [O III] sources in Fig. 2 with a circular Gaussian function that is meant to represent the shape of the PSF of our observations. The full-width at half maximum (FWHM) of such a PSF was first measured by matching the most cleanly detected of our sources (corresponding to source 21 of Ciardullo et al. 1989), and subsequently held constant when measuring the flux of other known or candidate PNe. The Gaussian model was resampled in SAURON $0 .{ }^{\prime \prime} 8 \times 0$.' 8 bins before comparing it to the data, and for close PN sources care was taken to deblend their fluxes by fitting simultaneously two or more Gaussian functions. Based on these fits, we deemed a given source of [O III] emission a detected $\mathrm{PN}$ if $A / r N>3$ within at least the FWHM of the Gaussian model. The model itself was then used to measure the [O III] flux of the PN, by integrating the entire Gaussian function out to radii where otherwise the [O III] emission generally would not be detected or could come from other nearby PNe.

According to the previous detection criterion we find $13 \mathrm{PNe}$ in the central regions of M32 observed by SAURON, with two additional sources that barely miss the detection threshold due to just 

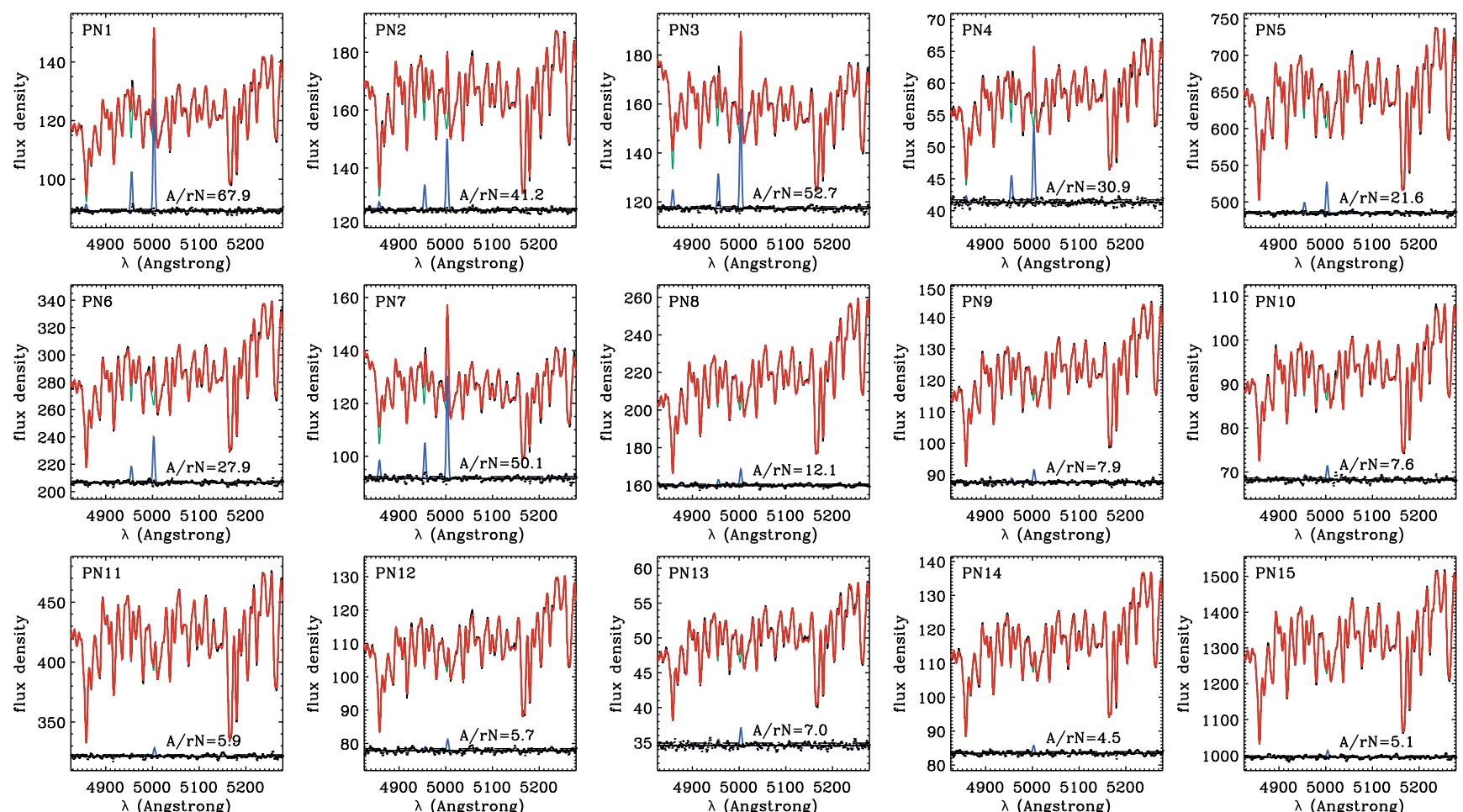

Figure 5. SAURON aperture spectra for the PNe sources shown in Fig. 3 along with our best fit for the PNe emission and the background stellar continnum. Such spectra were extracted within a radius $r=3 \sigma_{\mathrm{PSF}}$ from the center of the best-fitting Gaussian models plotted in Fig. 4 and correspond to the sum of all the SAURON spectra observed in the bins shown in each of the panels of that figure. In each of the panels shown here the red line shows the sum of the stellar and nebular model (green and blue lines), with flux densities in $10^{-16} \mathrm{erg} \mathrm{s}^{-1} \mathrm{~cm}^{-2} \operatorname{arcsec}^{-2} \AA^{-1}$, whereas the small points show the fit residuals around a zero level that has been rescaled for clarity. The values of the $A / r N$ ratio for the [O III] $\lambda 5007$ line is printed at the bottom of each panel, and show that the [O III] emission is detected in each spectrum, including those extracted around the doubtful PN sources 14 and 15.

one value of $A / r N$ being less then 3 within the FWHM of the models, which turned out to be $1^{\prime \prime} .94$ for a $\sigma_{\mathrm{PSF}}=00^{\prime \prime} 82$. Each of these sources is identified and labelled in Fig. 3 which maps the [O III] flux observed in M32 and shows with red contours the best fitting Gaussian models to each of the detected PNe, including the questionable sources with dotted lines. The fit to the [O III] flux distribution of these sources can be better appreciated in Fig. 4 where the radial profile for the [O III] flux of each PN is plotted together with its best-fitting Gaussian model. In Fig. 4 the [O III] data points are colour-coded according to the value of the $A / r N$ ratio measured in each bin to illustrate that down to our [O III] detection threshold of $A / r N=3$ the [O III] fluxes follow very well the PSF model, which provides an independent check of our $A / r N$ detection criterion for the [O III] emission.

Fig. 4 also shows how for each source we determine the minimum flux that we could have measured at the particular position of the PN. In fact, we can expect such detection limits to vary across the galaxy, and more specifically to increase towards the center where only the brightest PNe lead to [O III] lines of sufficient strength to stick out above the higher level of statistical noise that comes with a brighter stellar continuum. Following our detection criterion for $\mathrm{PNe}$, we set the detection limit at the location of each our PN sources as the flux of the Gaussian model (shown with a black line in Fig. (4) that would match the [O III] fluxes if these, and the corresponding values of the [O III] $\lambda 5007$ line amplitude $A$, would be rescaled until the minimum $A / r N$ value inside a FWHM reaches the threshold of $A / r N=3$. As expected, for the doubtful sources the detection limits exceed the observed flux of the best model, since the minimum $A / r N$ value is already less than 3 and therefore the data have to be scaled up, instead of down (although by just $\sim 7 \%$ in both cases). The significance of the detection of our PNe sources determines the order in which the PNe are labelled in Figs. 3, 4 and subsequent figures.

To further illustrate the degree to which all the PNe shown in Fig. 3 are detected, for each source we added all the SAURON spectra within a radius $r=3 \sigma_{\mathrm{PSF}}$ from the center of the bestfitting Gaussian models (corresponding to all the bins plotted in the panels of Fig. 4), and fitted the resulting spectra with our standard procedure. Fig. 5 shows such aperture spectra and our best fit to both the nebular emission from each PN and to the stellar continuum observed along the line-of-sight towards them. We note that the $A / r N$ values for the [O III] emission measured in these spectra always indicate a detection, even within a $3 \sigma_{\mathrm{PSF}}$ aperture that is 6.5 times wider than the FWHM region where we decided to assess the detection of PNe and within which we might have expected the emission from the weakest of our PNe to be lost against a larger stellar background. That this is never the case, however, suggests that the two questionable sources might as well be regarded as marginal detections, which is why we will keep considering them in the remainder of the paper.

To conclude this section in Tab. 1 we list, for both firmly and barely detected $\mathrm{PNe}$ in $\mathrm{M} 32$, the position relative to the center of the galaxy, the total $F_{5007}$ flux of the [O III] emission with its corresponding detection limit, the velocity of each source, and, when the $\mathrm{H} \beta$ line was also detected, the average value of the $[\mathrm{O} \mathrm{III}] / \mathrm{H} \beta$ ratio. The latter two measurements are based on fits to spectra similar to 
Table 1. Basic Properties of the PNe in the Optical Regions of M32

\begin{tabular}{rrrrrrr}
\hline ID & $\begin{array}{r}\text { x-off } \\
(1)\end{array}$ & $\begin{array}{r}\text { y-off } \\
(2)\end{array}$ & $\begin{array}{r}F_{5007} \\
(3)\end{array}$ & $\begin{array}{r}F_{5007, \lim } \\
(5)\end{array}$ & $\begin{array}{r}V_{\mathrm{PN}} \\
(6)\end{array}$ & $\begin{array}{r}{[\mathrm{O} \mathrm{III]}] / \mathrm{H} \beta} \\
(7)\end{array}$ \\
\hline 1 & -4.48 & -15.50 & 136.7 & 7.6 & -214.2 & 11.5 \\
2 & -5.16 & 6.18 & 101.5 & 6.1 & -240.9 & 13.8 \\
3 & -8.13 & 1.91 & 96.3 & 6.9 & -190.2 & 6.3 \\
4 & 1.81 & -23.60 & 46.5 & 4.4 & -216.1 & 7.8 \\
5 & -0.74 & 3.86 & 152.3 & 14.5 & -260.0 & 13.3 \\
6 & -4.75 & -5.83 & 132.9 & 15.9 & -240.4 & 16.8 \\
7 & -9.75 & 1.84 & 76.2 & 10.2 & -225.6 & 7.7 \\
8 & 5.99 & -3.29 & 29.9 & 6.4 & -188.9 & - \\
9 & 9.78 & -6.45 & 13.1 & 5.6 & -188.1 & - \\
10 & 12.18 & -4.38 & 9.4 & 6.0 & -147.5 & - \\
11 & -4.20 & -2.09 & 16.7 & 11.3 & -155.6 & - \\
12 & -11.33 & -9.69 & 15.1 & 11.2 & -133.6 & - \\
13 & 8.92 & -20.59 & 9.1 & 8.2 & -189.1 & - \\
14 & 4.08 & 13.19 & 7.9 & 8.5 & -231.6 & - \\
15 & 1.15 & -1.62 & 52.6 & 56.6 & -148.1 & - \\
\hline
\end{tabular}

Notes: (1) PN ID. (2)-(3) R.A. and Declination offset position, in arcseconds, from the center of M32. (4)-(5) Total [O III] 25007 flux and detection limit, in $10^{-16} \mathrm{erg} \mathrm{s}^{-1} \mathrm{~cm}^{-2}$. (6) Velocity in $\mathrm{km} \mathrm{s}^{-1}$, as measured in spectra extracted within a FWHM wide aperture around the center of the best-fitting Gaussian models shown in Fig. 3 (7) $[\mathrm{O} \mathrm{III]/H} \beta$ in the same spectra, when $\mathrm{H} \beta$ is detected. As shown in Fig. 1 our sources 1 to 7 were already detected by Ciardullo et al. (1989) who, in the order, labelled them with the ID of 21, 26, 24, 20, 27, 23 and 25 .

those presented in Fig. 5, but now extracted within a FWHM-wide aperture (rather than within a radius $r=3 \sigma_{\mathrm{PSF}}$ ) in order to maximise the emission-line signal and better isolate the kinematics of $\mathrm{PNe}$ that are close to each other in projection.

\section{RESULTS}

The analysis described in the previous section has delivered the solid detection of $13 \mathrm{PNe}$ within the optical regions of M32, with an additional 2 sources where the observed [O III] flux distribution is only marginally consistent with the emission from an unresolved PN. Given the systemic velocity $V_{\text {sys }}=-197 \mathrm{~km} \mathrm{~s}^{-1}$ of M32 and its average stellar velocity dispersion within one effective radius $\sigma_{e}=60 \mathrm{~km} \mathrm{~s}^{-1}$ (Cappellari et al. 2006), the velocities listed in Tab. 1 show that the observed PNe very likely belong to M32, rather than to the disk or halo of M31 where PNe move on average at a speed of $-400 \mathrm{~km} \mathrm{~s}^{-1}$ (Merrett et al. 2006). In fact, the case for membership in M32 holds even at a local level, when the PNe velocity $V_{\mathrm{PN}}$ is compared with the mean stellar velocity $V_{\star}$ and velocity dispersion $\sigma_{\star}$ measured along the line-of-sight towards their location. Using FWHM-wide aperture spectra to better separate the velocity $V_{\mathrm{PN}}$ of blended or close PNe and larger $3 \sigma_{\mathrm{PSF}}$-wide apertures to extract robust $V_{\star}$ and $\sigma_{\star}$ measurements also in the outskirts of M32, we found that only in 3 cases $\left|V_{\mathrm{PN}}-V_{\star}\right|>\sigma_{\star}$, even less often than what is normally expected for 15 sources. Tab. 1 also lists, when we could measure it, large values for the $[\mathrm{O}$ III $] / \mathrm{H} \beta$ ratio, thus showing that these are indeed high-ionisation sources most likely consistent with PN spectra. Finally, we note that for the PNe that were also detected by Ciardullo et al. (1989) our flux values agree fairly well, within a few percent, with the values reported in that work. For instance, for the brightest PN in our sample (source 5) we measure a $F_{5007}$ flux that is only $8 \%$ fainter than what was found by Ciardullo et al. (1989, source 27 in their Tab. 7).

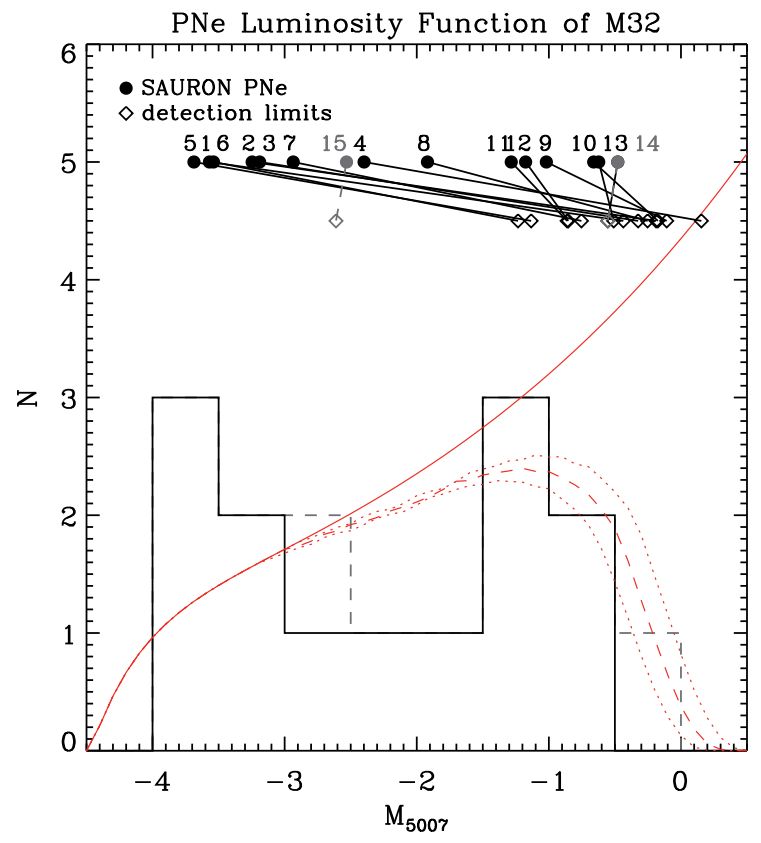

Figure 6. Luminosity function of the PNe sources observed with SAURON in the optical regions of M32, including marginal detections (dashed gray histogram) and along with the theoretical form of the PNe luminosity function (red solid line, from Ciardullo et al. 1989). The absolute magnitude of each PNe is shown at the top of the figure with filled circles that are plotted at an arbitrary constant ordinate and that are connected to open diamonds showing the magnitude corresponding to the detection limit of each source (with marginal sources shown in grey). The dashed line shows the PNe luminosity function multiplied by the median values of the completeness function across the entire SAURON field, whereas the dotted lines indicate the range by which the expected number of detected PNe would vary depending on their exact position within the $0 .{ }^{\prime \prime} 8 \times 0$.' 8 SAURON bins (see text and Fig.7for details). By integrating such a completeness-corrected luminosity function we obtain the total number of PNe that we would expect to detect within the region mapped by SAURON, and by matching this value with the actual number of observed PNe, 15 including marginal detections, we obtain our best estimate for the normalisation of the PNe luminosity function of M32. At first sight, the observed PNLF would look inconsistent with the theoretical prediction even accounting for incompleteness, in particular at its brightest end. A Kolgomorov-Smirnov test and the simulations shown in Fig. 8 demonstrate however that such a discrepancy can be expected with only 15 objects.

Fig. 6 presents the luminosity function of the PNe found in the optical regions of M32 by our SAURON observations, which essentially map this galaxy within its effective radius $R_{e}=30^{\prime \prime}$. To construct such a PNLF we followed the definition of Ciardullo et al. (1989) to compute the apparent V-band magnitude $m_{5007}=$ $-2.5 \log F_{5007}-13.74$ of our sources, and derived the values for the absolute magnitude $M_{5007}$ that are shown in Fig. 6 by adopting a distance modulus of 24.49 magnitudes. This corresponds to a distance of $791 \mathrm{kpc}$, and it is the same value based on the surfacebrightness fluctuation measurements of Tonry et al. (2001) that was used by Cappellari et al. (2006).

Fig. 6 also shows with a red line the expected form for the PNLF introduced by Ciardullo et al., who combined the simple Henize \& Westerlund (1963) model for a population of slowly fading and expanding PN envelopes with a sharp exponential cutoff at the high-mass end. The shape of our observed PNLF is inconsistent with such a theoretical curve, but this is hardly surprising 

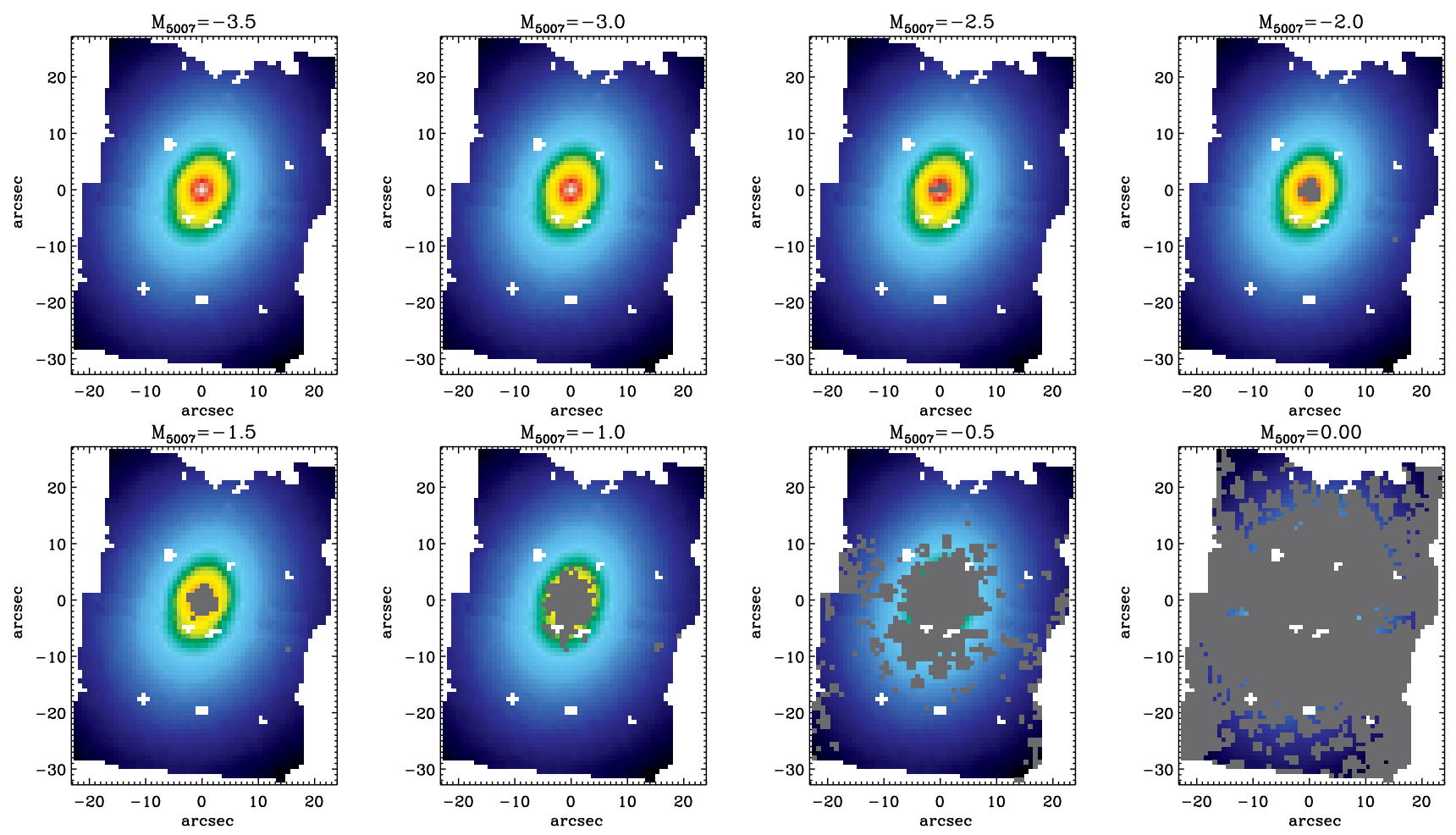

Figure 7. Reconstructed optical images of M32, in logarithmic flux scale, showing with grey bins the regions where, from left to right, PNe of increasing absolute magnitude $M_{5007}$ and decreasing brightness would not be detected. To check whether in a given bin of the SAURON field a PN of absolute magnitude $M_{5007}$ would be detected, we generated at that position a Gaussian model for the [O III] flux of a PN of that brightness in M32, deriving also the corresponding spectral density values for the amplitude of the [O III] line. Using the values for the level of the noise $r N$ in the residuals of our spectral fits we then computed the values of the $A / r N$ ratio around the PN position and, using the criterion introduced in $\delta 3.2$ simply checked whether $A / r N>3$ within a FWHM from the centre of the Gaussian model, which in these particular simulations correspond to the center of the SAURON bins.

considering that at the faint end our PN number counts are affected by incompleteness. To a first approximation the onset of this bias can be appreciated by noticing in Fig. 6 how the values for the absolute magnitude corresponding to the detection limits of each of our sources (shown with open diamonds) pile up from a $M_{5007} \sim-1.5$ and brighter, corresponding to an apparent magnitude limit of $m_{5007} \sim 23$. In general, while looking at bigger galaxies than M32 with a correspondingly larger number of PNe or at very close objects such as the large and small Magellanic clouds, other studies use the PNe that are found within the completeness limit in order to determine both the best normalisation for the theoretical PNLF and to test whether this could in fact be considered the parent distribution for the data. In the case of M32, however, there are simply not be enough PNe within the completeness limit (say for $M_{5007}<-2$, taking a conservative guess from Fig. 6) to confidently carry out such a measurement and test. We therefore decided to first understand how our observational biases would affect the theoretical form of Ciardullo et al. for the PNLF, to then test whether all our data, over the entire $M_{5007}$ range, could have been drawn from the corresponding completeness-corrected model PNLF, while also determining the best normalisation for it.

Fig. 7 presents simulations designed to show how we derive the completeness function of our observations, that is, the probability as a function of absolute magnitude $M_{5007}$ that a PN of that brightness could be detected across the entire field-of-view of the SAURON observations for M32. Since at any given luminosity the number of expected $\mathrm{PNe}$ scales with the total number of stars, such a probability is just the fraction of the total stellar flux encompassed by our field-of-view that is observed within the area over which PNe of a given absolute magnitude $M_{5007}$ could be detected. To then decide whether a PN of given $M_{5007}$ could be detected at any given position, we generated a Gaussian PN model of total flux corresponding to the apparent magnitude $m_{5007}$ and, using the values of $r N$ obtained from our spectral fits across the entire field-of-view, simply checked whether $A / r N>3$ within a FWHM of the Gaussian model. As Fig. 7 illustrates, starting from an absolute magnitude of $M_{5007} \sim-2.5\left(m_{5007} \sim 22\right)$ it becomes more and more difficult to detect PNe near the central region of the galaxy, with the area of non-detectability (shown with grey bins) quickly expanding outward for PNe fainter than $M_{5007} \sim-1.0$ $\left(m_{5007} \sim 23.5\right)$. More specifically, we are $100 \%$ complete down to $M_{5007} \sim-3$, whereas we lose about $5 \%$ of PNe with an absolute magnitude of $M_{5007} \sim-2.5$ and nearly $26 \%$ of those with $M_{5007} \sim-1.0$. Fig. 7 shows simulations for just eight $M_{5007}$ values and while placing the Gaussian PN models at the center of each SAURON $0 . .8 \times 0$. . 8 bin, but to derive the completeness function and the uncertainties associated with it we adopted a more refined grid of $M_{5007}$ values and accounted for the impact of randomly placing the Gaussian models within the SAURON bins.

When the theoretical PNLF of Ciardullo et al. is multiplied by the completeness function that we have just derived, we finally obtain the completeness-corrected prediction for the expected number of PNe in M32 that is shown by the red dashed curve in Fig.6 with the dotted red lines tracing the scatter in this correction due to the exact PNe position in the SAURON bins. A KolgomorovSmirnov test reveals that this corrected PNLF can be regarded as 
Sarzi et al.

the parent distribution for our data, since there is a $82 \%$ probability that all of our $15 \mathrm{PNe}$ could have been drawn from it, and a $52 \%$ probability when considering only the 13 secure PNe. By integrating the completeness-corrected luminosity function we obtain the total number of PNe that we would expect to detect, and by matching this value with the actual number of observed $\mathrm{PNe}$, 15 including marginal detections, we obtain our best estimate for the normalisation of the PNe luminosity function of M32 within the region mapped by our SAURON observations. Such a normalisation is generally expressed in terms of the luminosity-specific number density of PNe, through the ratio $\alpha=N_{\mathrm{PNe}} / L_{\mathrm{bol}}$ between the total expected number of PNe $N_{\mathrm{PNe}}$ in a stellar population and the total bolometric luminosity $L_{\mathrm{bol}}$ of the latter. Typically, the total number of $\mathrm{PNe}$ is estimated by integrating the normalised PNLF of Ciardullo et al. from the brightest observed absolute magnitude $M_{\star, 5007}=-4.47$ to 8 magnitudes fainter, which is the limit where Henize \& Westerlund (1963) locate the faintest PNe. Yet, since the faint end of the PNLF has not been well constrained by observations, in some instances $\alpha$ is provided only within the completeness limit and such an extrapolation is avoided. Most often in the literature this limit lies within 2.5 magnitudes of $M_{\star, 5007}$ and the luminosity-specific number density parameter is therefore indicated as $\alpha_{2.5}$. Adopting our normalisation there should be $76 \mathrm{PNe}$ in total within the central region of M32 mapped by SAURON and between 7 and 8 PNe (7.6) with $M_{5007}-M_{\star, 5007} \leqslant 2.5$. Given that our observations cover M32 within essentially its effective radius $R_{e}$ from I-band images, we are here encompassing nearly half of the total I-band luminosity, i.e. $L_{I}=2.3 \times 10^{8} M_{\odot}$ (see Cappellari et al. 2006, for $R_{e}$ and total luminosity I-band measurements). Adopting the reddening corrected $B-V=0.88$ colour of Buzzoni, Arnaboldi, \& Corradi (2006) for M32, and using Bruzual \& Charlot (2003) models for a Salpeter IMF and Solar metallicity, we then obtain a $B-I=2.01$ and thus a B-band luminosity of $L_{B}=1.3 \times 10^{8} M_{\odot}$. Finally, following Buzzoni, Arnaboldi, \& Corradi (2006) also for the bolometric correction to the B-band luminosity we arrive at a bolometric luminosity $L_{\mathrm{bol}}=3.4 \times 10^{8} M_{\odot}$, which means that for the optical regions of M32, within $R_{e}$, the luminosity-specific PN number density is $\alpha=2.2 \times 10^{-7} \mathrm{~L}_{\odot}^{-1}$, and a factor ten less for $\alpha_{2.5}$. Considering a $33 \%$ statistical error associated with our normalisation based on 15 points (for an intrinsic number of $25 \mathrm{PNe}$ within our detection magnitude range), these values are remarkably consistent with thefindings of both Buzzoni, Arnaboldi, \& Corradi (2006) and Ciardullo et al. (1989) who give $\alpha=1.70 \times 10^{-7} \mathrm{~L}_{\odot}^{-1}$ and $\alpha_{2.5}=2.23 \times 10^{-8} \mathrm{~L}_{\odot}^{-1}$, respectively.

To conclude, we have detected $15 \mathrm{PNe}$ in the optical regions of M32 that were mapped by our SAURON observations, and thanks to the integral-field nature of our data and by carefully accounting for the incompleteness of our observation we have been able not only to confirm kinematically that these sources belong to M32 but also that their observed luminosity function and their total number are consistent with the generally adopted shape of the PNLF (that of Ciardullo et al. 1989) and the typical values for the PNe number density $\alpha$ in early-type galaxies (a few $10^{-7}$ per $\mathrm{L}_{\odot}$, Buzzoni, Arnaboldi, \& Corradi 2006). Considering that our 600slong SAURON observations at the $4 \mathrm{~m}$ William Herchel Telescope collected nearly as many photons as the 3600s- and 1800s-long narrow-band observations of Ciardullo et al. (1989) at the $0.9 \mathrm{~m}$ and $2.1 \mathrm{~m}$ Kitt Peak telescopes, respectively, the fact that we more than doubled the number of known PNe in the central regions of M32 while reaching 5 times fainter [O III] PNe fluxes (see Tab1) illus- trates well the power of integral-field spectroscopy to study PNe in the optical regions of galaxies.

\section{DISCUSSION}

It is interesting to comment on our PN results in light of what is known about the central stellar population of M32 and the current explanations for both the shape and normalisation of the PNLF.

Rose et al. (2005) have shown that the luminosity-weighted mean stellar population of M32 at $1 R_{e}$ is older by $\sim 3 \mathrm{Gyr}$ and more metal-poor by about $-0.25 \mathrm{dex}$ in $[\mathrm{Fe} / \mathrm{H}]$ than the central value of $\sim 4 \mathrm{Gyr}$ and $[\mathrm{Fe} / \mathrm{H}] \sim 0.0$, which are trends that conspire to produce the flat color profiles previously reported in this object (Peletier 1993; Lauer et al. 1998). Episodes of star formation may enhance for a relative short period of time the bright-end of the PNe luminosity function of a galaxy, since massive main-sequence stars tend to produce also massive and bright central PNe stars (see e.g., the case of M33 in Ciardullo et al. 2004). For instance, Marigo et al. (2004) shows that stars with initial mass between 2.5 and $3.5 \mathrm{M}_{\odot}$ evolve to central PN stars of about $0.70-0.75 \mathrm{M}_{\odot}$ that can power nebular fluxes corresponding to the typical PNLF cut-off magnitude $M_{\star, 5007}$ of -4.47 . Since massive stars are short-lived, however, such a PNLF enhancement would be pretty weak already 3 Gyr after star formation ceased, and it would no longer apply to the brightest PNe but to objects with $M_{5007} \sim-2.5$ (Marigo et al. 2004). It is thus not surprising, in particular given the small number of PNe at our disposal, that we do not see such a signature in our observed PNLF. If anything, there seems to be a lack of PNe around that intermediate $M_{5007}$ value.

On the other hand, and like in other early-type galaxies with no evidence of recent (less than 1-Gyr-old) star formation, the presence in M32 of bright PNe at a time when the progeny of massive stars have long disappeared is a puzzle that has not yet been fully solved. In fact, the need for a massive central star in the brightest $\mathrm{PNe}$, with mass above $\sim 0.70 M_{\odot}$, is a result that still holds in the studies of Schönberner et al. (2007) and Méndez et al. (2008) that supersede the initial investigation of Marigo et al. (2004). One possible way to form the required high-mass PN cores, suggested by Ciardullo et al. (2005), involves the binary coalescence during their hydrogen burning phase of $\sim 1 \mathrm{M}_{\odot}$ stars, a process that has been associated with the formation of blue-stragglers and which could be facilitated in early-type galaxies by the abundance of $\sim 1 \mathrm{M}_{\odot}$ stars in old stellar systems. If such a second path to the formation of PNe exists, then we may also expect to find a PNLF characterised by a peak at high $M_{5007}$ above an otherwise monothonically increasing PNLF, corresponding to a standard Henize \& Westerlund population of PNe powered by only old central PN stars of relatively low mass. In light of this scenario, it would be tempting to see a bimodality in our observed PNLF (Fig. 6), which happens to show two peaks around $M_{5007} \sim-3.5$ and -1 that correspond to parent stellar masses near 2 and $1 \mathrm{M}_{\odot}$, respectively. However, due to small number statistics we cannot read too much in the observed shape of the M32 PNLF, as it is instructive to see through simple simulations such as those presented in Fig. 8 , which confirm the formal consistency indicated by a simple KS-test between our data and the completeness-corrected Ciardullo et al. form of the PNLF. Similarly, it would be difficult with our sample size to claim a significant difference in the kinematic behaviour of bright and faint PNe (as in NGC 4697, Sambhus, Gerhard \& Mendez 2006), which could occur for instance if the coalesced central stars of the PNe can form in globular clusters, as could 

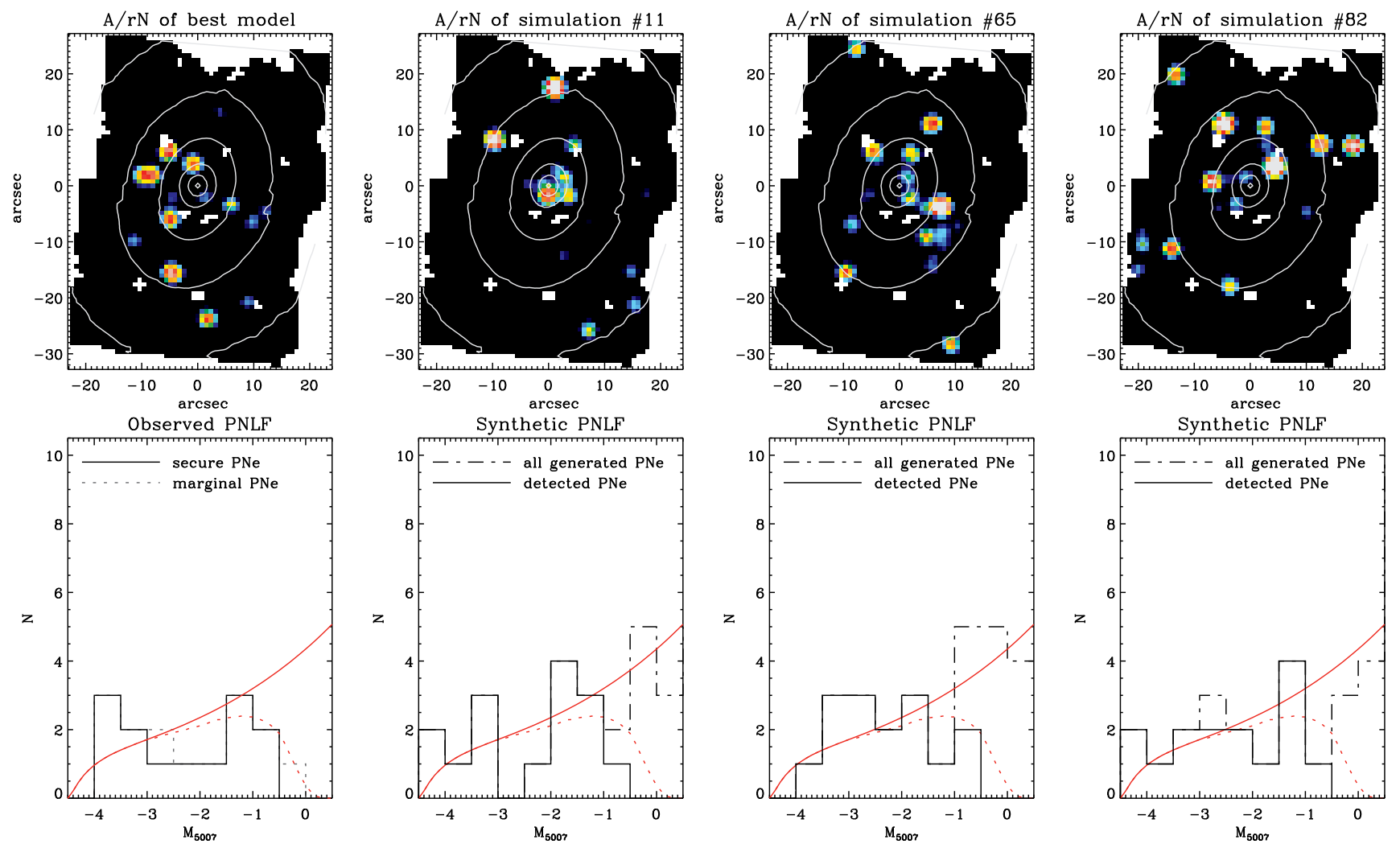

Figure 8. Comparison between the spatial distribution and the luminosity function of the PNe detected in the central region of M32 (top and lower left panels, respectively) and three particular synthetic realisations of the same quantities (central and right panels). More specifically, the top panels show only the spatial distribution of PNe that were or would be detected in the optical regions of M32 by displaying values for the $A / r N$ ratio above the $A / r N>3$ detection threshold, and which correspond (adopting the observed values of the residual-noise level $r N$ ) to the flux distribution of either our best-fitting Gaussian models to the PNe of M32 (3.2) or to similar Gaussian flux profiles for randomly generated PNe. Starting from an intrinsic Ciardullo et al. shape of the PNLF (red curves in lower panels) and adopting a normalisation leading to match the number of observed PNe once the PNLF is corrected for incompletess (red dashed lines, see 44 , the synthetic PN fields were generated by considering at any particular position in SAURON field of view the probability of having a PN of a given luminosity. Such a probability function simply corresponds to the total intrinsic PNLF rescaled by the fraction of stellar light that is observed in the SAURON spatial bin that is being considered. The [O III] flux of the simulated PNe was then "observed" by obtaining maps for the $A / r N$ ratio and by applying the same detection criteria described in 3.2 These simulations illustrate that, given the low number of PNe expected in M32, not much can be read in the apparent bimodality of the PNLF that we observe nor in the fact that the PNe of M32 seems to all be on the same side of the galaxy. In fact, these three particular simulations yielded the same total number of detected PNe than we observe (15), but generally this is not the case and the PNLF of such synthetic fields appear to differ even more from the observed PNLF than shown by the chosen examples.

be the case for X-ray binaries (Sarazin. Irwin \& Bregman 2000; White. Sarazin \& Kulkarni 2002) and where indeed blue-stragglers are often found. Alternatively, and consistent with the hypothesis of Marigo et al. (2004), a different kinematics for the brightest PNe may be a sign of recent accretion of a younger and smaller galaxy (Mamon. Dekel. \& Stoehr 2005). Yet, integral-field data allow for a direct comparison with the stellar kinematics as a function of $M_{5007}$, such as shown for M32 in Fig. 9 that would be interesting to apply to more massive systems hosting a larger number of PNe.

From a theoretical perspective, our best normalisaton of the PNLF in the optical regions of M32, with a value for the specific PN number density $\alpha$ in line with that of other early-type galaxies, also poses a problem. Indeed, as long as the time-scale for PN visibility depends mostly on the lifetime of their post-AGB cores (pAGB), then since cores of lower mass spend more time in their high-temperature regime $\alpha$ should increase with the stellar population age, whereas in fact quite the opposite is observed (see Buzzoni, Arnaboldi, \& Corradi 2006, for a comprehensive review of this discrepancy). A possible solution to this puzzle is to assume that a considerable fraction of the stellar population of earlytype galaxies ends up in the extreme hot end of the helium-burning horizontal-branch (HB), and that when such stars of very small hydrogen-envelope mass subsequently leave the HB they not only skip entirely their AGB phase (thus the name of AGB-manqué) but they also fail to produce a PN. As the AGB-manque evolution can effectively transfer some fraction of the pAGB energy budget to the integrated UV flux of the galaxy, this scenario could also explain the anticorrelation between the strength of the UV-upturn (as defined by Burnstein et al. 1988) and the luminosity-specific PN number density of early-type galaxies, which respectively increase and decrease with both galaxy mass and metallicity. In fact, it is in M32 that Brown et al. (2000) provided some support to this picture, finding with near-UV Hubble Space Telescope (HST) observations that the relative faint UV flux of M32 originates from hot HB stars and their AGB-manqué progeny rather than from their brighter postAGB counterparts. Yet, given the relatively long UV-bright lifetime of stars leaving the hot end of the $\mathrm{HB}\left(\sim 10^{6}-10^{7} \mathrm{yrs}\right.$ compared 


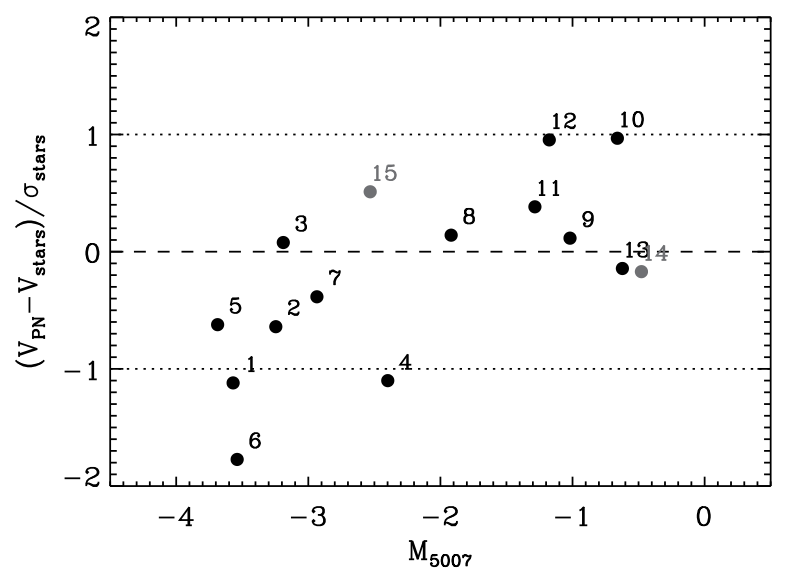

Figure 9. Velocity $V_{\mathrm{PN}}$ of the PNe in M32 relative to the average stellar velocity $V_{\star}$ along the line-of-sight towards the $\mathrm{PNe}$ position and normalised by the stellar velocity dispersion $\sigma_{\star}$ in the same direction, sorted by their absolute magnitude $M_{5007}$. PNe sources are labelled and colour-coded as in Fig 6 The dotted horizontal lines for $\left|V_{\mathrm{PN}}-V_{\star}\right| / \sigma_{\star}=1$ delineate the region within which there is a $68 \%$ probability that a given $\mathrm{PN}$ belongs to the stellar population of M32, assuming a Gaussian stellar line-of-sight velocity distribution. The $V_{\mathrm{PN}}$ values are plotted against $M_{5007}$ of the $\mathrm{PNe}$ to check whether bright and faint $\mathrm{PNe}$, possibly powered by central stars having evolved via distinct binary and single-star channels, have different kinematics. A Spearman rank coefficient of 0.6 suggests a correlation in this figure, although this would not be dramatically significant since there is still a $2 \%$ probability that the plotted quantities follow each other monotonically only by mere chance.

to $\sim 10^{3}-10^{4}$ yrs for stars leaving the HB red end) Brown et al. (2000) conclude that only a small fraction of HB stars in M32 live at its blue tip, so that in fact many more bright pAGB should be observed. The recent analysis of the UV colour-magnitude diagram (CMD) of M32 by Brown et al. (2008) further reinforces this result. The findings of Brown et al. (2000, 2008) not only contrast again with the notion that only hot and less massive HB stars should now form in the old stellar population of M32 (unless one considers binary coalescence), but also add an additional problem for the current models of the late evolution of stars.

To explain such a dearth of pAGB stars, among other possibilities Brown et al. (2000) suggested that pAGB could be obscured by circumstellar material, in particular as they cross the UV CMD before reaching the region where they peak in temperature at around $60,000 \mathrm{~K}$. This is an interesting possibility considering that $\mathrm{PNe}$ are themselves affected by dust extinction and given that, assuming a Cardelli, Clayton, \& Mathis (1989) reddening law and $\mathrm{R}_{\mathrm{V}}=3.1$, the typical extinction of 0.7 magnitude at $5007 \AA$ for $\mathrm{PNe}$ in both star-forming and old galaxies (Herrmann \& Ciardullo 2009; Richer, Stasińka, \& McCall 1999; Jacoby \& Ciardullo 1999) would translate into considerable reddening values of $\sim 1.71$ and $\sim 1.67$ in the HST far- and near-UV passbands (FUV and NUV), respectively. To actually check the impact of reddening in the pAGB population of $\mathrm{M} 32$, or at least for the fraction that is presently powering a PN, we looked in the catalogue of Brown et al. (2008, kindly provided by $\mathrm{T}$. Brown) for the best candidate central star for our PNe sources and plotted their position in the UV CMD shown in Fig. 10 The field-of-view of the Brown et al. HST observations falls entirely within the area covered by our SAURON data, being $24^{\prime \prime} \times 24^{\prime \prime}$ and only slightly offset from the center of M32, but un-

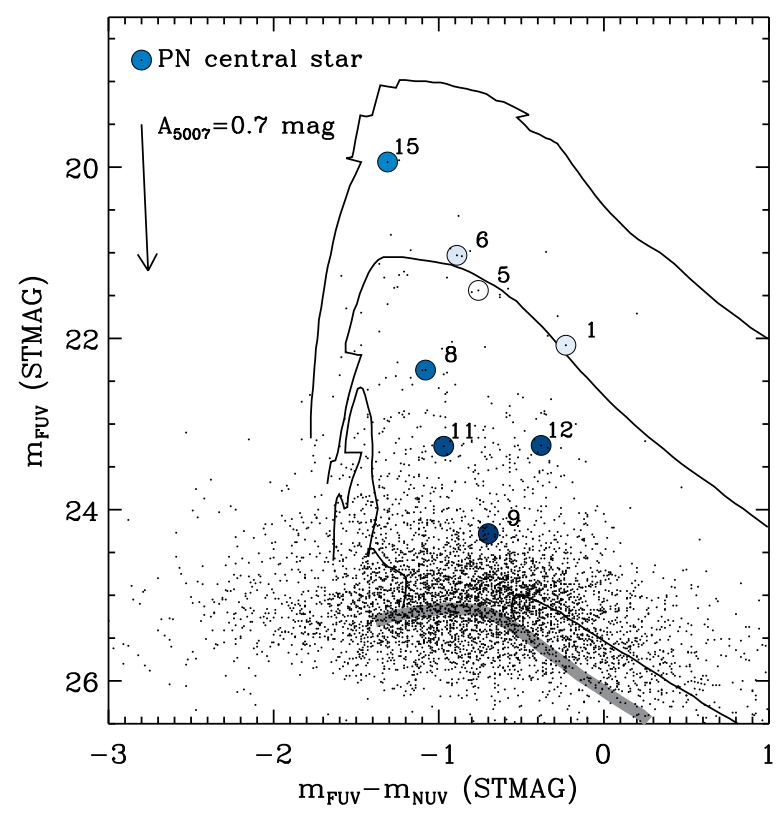

Figure 10. UV colour-magnitude diagram (CMD) for the HST data of Brown et al. 2008) with solid lines tracing, from top to bottom, the evolutionary path of pAGB, early-pAGB, and AGB-manqué stars starting from the horizontal branch (shown by the grey thick line) and ending in the region occupied by white dwarves. The asymptotic giant branch, from which pAGB and early-pAGB stars cross back the UV CMD is to the left and outside the plotting range. The large circles indicate the location in this CMD of the brightest and closest UV sources to the PNe falling within the fieldof-view of the Brown et al. observations. Such PN central star candidates are colour-coded (from dark blue to white) according to the brightness of the PN they could be powering. Assuming a Cardelli, Clavton, \& Mathis (1989) reddening law, the arrow at the top right of the figure shows the impact of dust reddening in the far- and near-UV HST passbands for an extinction of 0.7 magnitude at $5007 \AA$. This reddening value for PNe is typically observed in both star-forming and old galaxies, and would suffice to bring the candidate central stars of the PNe in the optical regions of M32 close to the location where pAGB and early-pAGB stars cross the UV CMD before reaching the WD cooling curve, to the left of the bluer end of the horizontal branch.

fortunately only 8 of our PNe end up within it. As we are looking to assess as conservatively as possible the impact of reddening on pAGB stars, in Fig. 10 we have located not only the closest but also the brightest of the UV stars near the location of our PNe. Except for PN 9, we could always find a pretty unique UV star within a radius of 0.97 (half a FWHM) with a far-UV magnitude that would stick out from the distribution of magnitude values of all stars within 2 ". 5 ( $\left.3 \sigma_{\mathrm{PSF}}\right)$ of our PN by at least 3 times the scatter in such values. Furthermore, except for PNe 12 and 15, such bright UV stars are always quite close to our PNe, within 0.'25. For PN 15 the relative large distance of the candidate star ( $\left.0^{\prime \prime} 63\right)$ is less of a concern, given that admittedly our Gaussian model does not fully reproduce the [O III] flux distribution of this source, but for PN 12 $(0.85)$ it is possible that the central PN star lies elsewhere near the PN and therefore also in the CMD of Fig. 10 This is also most certainly the case of PN 9, for which the plotted position in Fig. 10 should be considered as upper limit for the far-UV brightness of its central star. It is important to note, however, that the central stars of even relatively faint $\mathrm{PNe}$ such as $\mathrm{PN} 9$ and 12 should still be intrinsically fairly bright pAGB or early-post AGB stars (epAGB) and, 
in particular, that our PNe could not be powered by stars that have already reached the white-dwarf (WD) cooling curve. Indeed, the models of Marigo et al. (2004, see their Fig. 10) shows that at that stage it is possible to obtain only PNe of $M_{5007}>0.5$, which are much fainter than the PNe that we can detect in M32. On the other hand, even considering the uncertain position of PN 9 and possibly PN 12 in Fig. 10, we note that accounting for the typical reddening observed in PNe would bring back up and left the position of the central PN candidate stars of our brightest and faintest PNe near to the location in the UV CMD where pAGB and epAGB reach their peak temperature and spend most of their time, for FUV magnitudes around $\sim 20$ and $\sim 22$, respectively, and a FUV-NUV colour $\sim-1.5$. In fact, we note that PNe as bright as our PN 1, 5 and 6 , with $M_{5007}<-3.5$, can only be powered by central stars that came from main-sequence stars of masses between 1.6 and $2.5 \mathrm{M}_{\odot}$ and which would cross the UV CMD of Fig. 10 along the brightest pAGB tracks (again, see Marigo et al. 2004), which implies that these sources actually must be reddened.

We can double-check this result by using our integral-field data to estimate the position in the theoretical $\log T_{\text {eff }}-\log L$ Hertzsprung-Russell (HR) diagram of the PNe for which we can also detect the $\mathrm{H} \beta$ emission, and then compare their location to the evolutionary tracks of pAGB stars. Through the models of Dopita, Jacoby, \& Vassiliadis (1992) it is indeed possible to estimate the temperature $T_{\text {eff }}$ and luminosity $L$ of the PNe central star using the value for the $[\mathrm{O} I \mathrm{III}] / \mathrm{H} \beta$ ratio and the $\mathrm{H} \beta$ flux, respectively. These models predict a conversion efficiency $\epsilon$ between the bolometric luminosity $L$ of the central star and the $\mathrm{H} \beta$ recombination flux from the PN that is rather constant and independent of the gas temperature $T_{\mathrm{e}}$ or metallicity $Z_{\text {gas }}$. On the other hand, since Oxygen is the main coolant of PNe, the [O III] $\lambda 5007$ flux depends eavily on $T_{\mathrm{e}}$ and $Z_{\text {gas }}$ and some knowledge of these quantities further helps constraining $T_{\text {eff }}$ with the $[\mathrm{O}$ III $] / \mathrm{H} \beta$ ratio. The restricted wavelength range of our SAURON data does not grant us access to the weak [O III] $\lambda 4363$ line that is normally used to measure $T_{\mathrm{e}}$, but we can use our spectroscopic data to measure the stellar metallicity along the line-of-sight of our PNe and thus gauge the metallicity of their central star, which in turn relates naturally to $Z_{\text {gas }}$.

There are $7 \mathrm{PNe}$ in the central regions of M32 for which we could measure the $\left[\mathrm{O} \mathrm{III}_{\mathrm{II}}\right] \mathrm{H} \beta$ ratio (Tab. 1 , with values ranging from to just above 6 to almost 17. The SAURON aperture spectra around these objects (extracted within a radius $r=3 \sigma_{\mathrm{PSF}}$, Fig. 5] indicate values for the $\mathrm{H} \beta$ absorption line and the [MgFe50]' index of Kuntschner et al. (2010) between $1.77-1.92 \AA$ and 3.01 - $3.08 \AA$, respectively, which in turn yield an estimate for the stellar metallicity $[\mathrm{Z} / \mathrm{H}]$ between -0.4 and -0.33 (see Fig. 3 of Kuntschner et al. 2010), depending on whether one adopts the models of Schiavon (2007) or of Thomas, Maraston, \& Bender (2003). With this gauge for the central star metallicity we can infer from Fig. 3 of Dopita, Jacoby, \& Vassiliadis a star temperature $T_{\text {eff }}$ around $\sim 55,000 \mathrm{~K}$ for the PNe with low $[\mathrm{O} \mathrm{III}] / \mathrm{H} \beta$ values $\sim 6-$ 8 , and above $\sim 85,000 \mathrm{~K}$ for the PNe with $\left[\mathrm{O}_{\mathrm{III}}\right] / \mathrm{H} \beta>11$. From Fig. 1 of Dopita et al. this range of temperatures would then correspond to an average conversion efficiency $\epsilon$ around $0.7 \%$, which translate our observed $\mathrm{H} \beta$ fluxes into central star luminosities in the $\log \left(L / L_{\odot}\right)=3.0-3.2$ range. These $T_{\text {eff }}$ and $L$ values would place the $7 \mathrm{PNe}$ with $[\mathrm{O} \mathrm{III}] / \mathrm{H} \beta$ measurements from our SAURON observations right below and to the right of the post-AGB evolutionary paths that are normaly used in the HR diagram to trace the luminosity evolution of PNe (see, e.g., Fig. 1 of Mendez et al. 2008 and Fig. 3 of Schonberner et al. 2010), too cold to be on the cooling track of white dwarves (which cannot power PNe) and too faint to be on par with the pAGB stars that normally produce PNe (which range in luminosity between $\log (L / L \odot)=3.5-4.0$ ). Assuming a Solar or nearly Solar metallicity for the central stellar population of M32, as found by Rose et al. (2005), would lead to an even more stringent result since in this case the central star $T_{\text {eff }}$ of the highly-excited PNe would be better constrained to lower values by the models of Dopita et al. Thus, also for this second subsample of $\mathrm{PNe}$ it would seem necessary to invoke dust reddening in order to to reconcile our data with the model expectations, further supporting the possible role of circumstellar dust in producing the apparent dearth of pAGB stars in M32.

\section{CONCLUSIONS AND FUTURE PROSPECTS}

Using SAURON data for the nearby elliptical M32 we have shown how, by means of a careful subtraction of the stellar background, integral-field spectroscopy (IFS) allows to detect the emission of single Planetary Nebulae (PNe) in the optical regions of early-type galaxies down to flux levels otherwise hardly accessible to standard narrow-band photometry. In turn, this makes it possible to trace the PNe luminosity function (PNLF) within a wider completeness limit and in galactic regions where stellar populations gradients can be the most extreme, and therefore where studying how the shape and normalization of the PNLF originate from the bulk of a galactic stellar population could be the most instructive. Moreover, we have shown that the incompleteness of a survey for PNe in the optical region of a galaxy can be very well understood when using IFS data, and that the possibility to extract also the stellar kinematics further allows to check the membership of $\mathrm{PNe}$ on a local basis, that is, along the line-of-sight towards a given PN. Such a local comparison between the PNe and stellar kinematics could be useful to identify subpopulations of PNe of different origins. Finally, although with the SAURON data we could only confirm the high-ionisation nature of nearly half of our sources, on a longer wavelength range IFS has a great potential to study the nebular spectrum of extragalactic $\mathrm{PNe}$, as already illustrated in the pioneering work of Roth et al. (2004).

In the specific case of M32, with just two 10-minutes-long SAURON pointings, we have doubled the number of known $\mathrm{PNe}$ within the effective radius of this galaxy and detected $\mathrm{PNe}$ five times fainter than previously found with narrow-band imaging by Ciardullo et al. (1989), while collecting slightly less the same number of photons. Furthermore, accounting for the incompleteness of our survey across the entire luminosity range spanned by our detected PNe, we have concluded that the central PNe population of M32 is consistent with the generally adopted shape for the PNLF (that of Ciardullo et al.) and its typical normalization observed in early-type galaxies (i.e. with a PNe number density $\alpha$ of a few $10^{-7}$ per $\left.\mathrm{L}_{\odot}\right)$. Finally, thanks to exquisite $H S T$ data and to the proximity of M32 we were able to combine our PNe measurements with images for the resolved UV-bright stellar population of M32 and conclude that the PNe central stars may be considerably reddened by dust, a result that we could double-check thanks to the IFS nature of our data by estimating the temperature and luminosity for the central star of the $\mathrm{PNe}$ with $[\mathrm{O}$ III $] / \mathrm{H} \beta$ measurements. This finding would support the suggestion by Brown et al. that circumnstellar dust extinction could explain the apparent dearth of pAGB stars in M32, a problem that may also apply to the halo of the Milky Way (Weston, Napiwotzki, \& Catalán 2010).

Given the intrinsically small number of PNe that is expected in the central regions of such a small galaxy as M32, it is unlikely that with the current instruments (e.g., SAURON or VIMOS) fur- 
ther IFS observations will reveal a sufficiently larger number of objects to add either to what found in this work or to what is already known in the outskirts of M32 from narrow-band imaging or slit-less spectroscopy, even though more IFS data could allow for more emission-line diagnostics.

On the other hand, there is a wealth of SAURON data for many more early-type galaxies that was aquired over the course of the SAURON (de Zeeuw et al. 2002) and ATLAS ${ }^{3 \mathrm{D}}$ (Cappellari et al. 2011) surveys where the presence of PNe have not yet been systematically investigated. In fact, even though a few PNe were detected in Sarzi et al. (2006), many more PNe have certainly been missed because a large fraction of the data were spatially binned in order to extract a reliable stellar kinematics and due to the common presence of diffuse ionised gas. Indeed spatial binning washes out the signal of the weaker PNe against the stellar background, whereas extended nebular emission makes it difficult to detect $\mathrm{PNe}$ unless their distinctive spectrum characterised by high-ionisation and narrow lines is purposely looked for. Paying attention also to the unresolved nature of the PNe emission and working with unbinned data using an educated guess for the stellar kinematics (for instance based on relatively simple but functional dynamical models, see Cappellari 2008) it may be possible to tap a significant PNe population in the central regions of nearby early-type galaxies. Indeed, although the SAURON and ATLAS ${ }^{3 \mathrm{D}}$ surveys have targeted much more distant objects than M32, the ability to carefully subtract the stellar background will still enable the detection of fainter $\mathrm{PNe}$ in the central regions of these galaxies compared to narrowband or slit-less spectroscopic surveys. Furthermore, the relatively large number of objects surveyed by these campaigns (260) will allow to combine the measurements from different galaxies, and for instance to better explore the bright end of the PNLF or variations of its shape and normalization as a function of the stellar age and metallicity that will also be provided by the these SAURON data.

To conclude, given the number of findings obtained here with a modest time investment (not even 30 minutes with overheads) on a $4 \mathrm{~m}$ telescope, it is exciting to consider what could be learned on the PNe population in the optical regions of other galaxies, when the next generation of integral-field spectrographs will be mounted on 8m-class telescopes, such as MUSE on VLT (see, e.g., Bacon et al. 2006). For instance, with a longer wavelength range and a larger light bucket, MUSE will not only find many more and fainter PNe, but it will also allow full diagnostic of their nebular spectrum that could lead to a direct measurement of the gas metallicity and to recognise other nebular sources that could pose as a $\mathrm{PNe}$ in narrow-band surveys (see, e.g., Frew \& Parker 2010). In turn the PNe metallicity could be compared with that of the underlying stellar population, which would also be better constrained thanks to an extended spectral range. Furthermore, the spatial resolution that will be provided to MUSE by the use of adaptive optics will allow further comparisons with HST or other space-based measurements for the resolved stellar population of nearby galaxies, such as those presented here for M32, which are bound to shed more light on the link between $\mathrm{PNe}$ and their parent stellar population.

\section{ACKNOWLEDGEMENTS}

MS is grateful to Thomas Brown, Paola Marigo, Harald Kuntschner, Ralph Napiwotzki and Brent Miszalski for the useful discussions, and to Thomas Brown for also providing the nearand far-UV HST data of M32. We are also indebted to the referee,
Robin Ciardullo, for his many suggestions. MS also acknowledges support from his STFC Advanced Fellowship (ST/F009186/1) and the Institut d'Astrophysique de Paris for its hospitality during part of the preparation of this paper, whereas MC acknowledges support from a STFC Advanced Fellowship (PP/D005574/1) and a Royal Society University Research Fellowship.

\section{REFERENCES}

Bacon R., et al., 2001, MNRAS, 326, 23

Bacon R., et al., 2006, The Messenger, 124, 5

Brown T. M., Bowers C. W., Kimble R. A., Sweigart A. V., Ferguson H. C., 2000, ApJ, 532, 308

Brown T. M., Smith E., Ferguson H. C., Sweigart A. V., Kimble R. A., Bowers C. W., 2008, ApJ, 682, 319

Bruzual G., Charlot S., 2003, MNRAS, 344, 1000

Burstein, D., Bertola, F, Buson, L. M., Faber, S. M., Lauer, T. R., 1988, ApJ, 328, 440

Buzzoni A., Arnaboldi M., Corradi R. L. M., 2006, MNRAS, 368, 877

Cardelli J. A., Clayton G. C., Mathis J. S., 1989, ApJ, 345, 245

Cappellari M., Copin Y., 2003, MNRAS, 342, 345

Cappellari M., Emsellem E., 2004, PASP, 116, 138

Cappellari M., et al., 2006, MNRAS, 366, 1126

Cappellari M., 2008, MNRAS, 390, 71

Cappellari M., et al., 2011, MNRAS, in press (doi:10.1111/j.1365-2966.2010.18174.x)

Ciardullo R., Jacoby G. H., Ford H. C., Neill J. D., 1989, ApJ, 339, 53

Ciardullo R., Durrell P. R., Laychak M. B., Herrmann K. A., Moody K., Jacoby G. H., Feldmeier J. J., 2004, ApJ, 614, 167

Ciardullo R., Sigurdsson S., Feldmeier J. J., Jacoby G. H., 2005, ApJ, 629, 499

Ciardullo R., 2006, IAUS, 234, 325

de Zeeuw P. T., et al., 2002, MNRAS, 329, 513

Dopita M. A., Jacoby G. H., Vassiliadis E., 1992, ApJ, 389, 27

Dopita M. A., et al., 1997, ApJ, 474, 188

Douglas N. G., et al., 2007, ApJ, 664, 257

Emsellem, E., et al. 2004, MNRAS, 352, 721

Falcón-Barroso, J., et al. 2006, MNRAS, 369, 529

Frew D. J., Parker Q. A., 2010, PASA, 27, 129

Jacoby G. H., Ciardullo R., Ford H. C., 1990, ApJ, 356, 332

Jacoby G. H., et al., 1992, PASP, 104, 599

Jacoby G. H., Ciardullo R., 1999, ApJ, 515, 169

Henize K. G., Westerlund B. E., 1963, ApJ, 137, 747

Herrmann K. A., Ciardullo R., 2009, ApJ, 703, 894

Lauer T. R., Faber S. M., Ajhar E. A., Grillmair C. J., Scowen P. A., 1998, AJ, 116, 2263

Kuntschner H., et al., 2010, MNRAS, 408, 97

Mamon G. A., Dekel A., Stoehr F., 2005, AIPC, 804, 345

Marigo P., Girardi L., Weiss A., Groenewegen M. A. T., Chiosi C., 2004, A\&A, 423, 995

Méndez R. H., Teodorescu A. M., Schönberner D., Jacob R., Steffen M., 2008, ApJ, 681, 325

Merrett H. R., et al., 2006, MNRAS, 369, 120

Peletier R. F., 1993, A\&A, 271, 51

Richer M. G., Stasińka G., McCall M. L. M., 1999, A\&AS, 135, 205

Romanowsky A. J., Douglas N. G., Arnaboldi M., Kuijken K., Merrifield M. R., Napolitano N. R., Capaccioli M., Freeman K. C., 2003, Sci, 301, 1696 
Rose J. A., Arimoto N., Caldwell N., Schiavon R. P., Vazdekis A., Yamada Y., 2005, AJ, 129, 712

Roth M. M., Becker T., Kelz A., Schmoll J., 2004, ApJ, 603, 531

Sambhus N., Gerhard, O., \& Mendez, R. H., 2006, AJ, 131, 837

Sánchez-Blázquez P., et al., 2006, MNRAS, 371, 703

Sarazin C. L., Irwin, J. A., \& Bregman, J. N., 2000, ApJ, 544, L101

Sarzi M., et al., 2006, MNRAS, 366, 1151

Sarzi M., et al., 2010, MNRAS, 402, 2187

Schiavon R. P., 2007, ApJS, 171, 146

Schönberner D., Jacob R., Steffen M., Sandin C., 2007, A\&A, 473, 467

Schönberner D., Jacob R., Sandin C., Steffen M., 2010, A\&A, 523, A86

Thomas D., Maraston C., Bender R., 2003, MNRAS, 339, 897

Tonry J. L., Dressler A., Blakeslee J. P., Ajhar E. A., Fletcher A. B., Luppino G. A., Metzger M. R., Moore C. B., 2001, ApJ, 546,681

Weston S., Napiwotzki R., Catalán S., 2010, AIPC, 1273, 197

White, R. E., Sarazin C. L., \& Kulkarni, S. R., 2002, ApJ, 571, L23 